\title{
The $\Lambda(1405)$ state in a chiral unitary approach with off-shell corrections to dimensional regularized loop functions
}

\author{
Fang-Yong Dong ${ }^{1}$, Bao-Xi Sun ${ }^{1,2}$ and Jing-Long Pang ${ }^{2}$ \\ ${ }^{1}$ College of Applied Sciences, Beijing University of Technology, Beijing 100124, China and \\ ${ }^{2}$ Department of Physics, Peking University, Beijing 100871, China
}

\begin{abstract}
The Bethe-Salpeter equation is solved in the framework of unitary coupled-channel approximation by using the pseudoscalar meson-baryon octet interaction. The loop function of the intermediate meson and baryon is deduced in a dimensional regularization scheme, where the relativistic kinetic effect and off-shell corrections are taken into account. According to the experimental data at the $K^{-} p$ threshold, the subtraction constants in the loop function are determined. The squared amplitude is suppressed strongly and only one $\Lambda(1405)$ state is generated dynamically in the strangeness $S=-1$ and isospin $I=0$ sector.
\end{abstract}

Keyword: Chiral Lagrangian, Kaon-baryon interaction, Hyperon

PACS numbers: 12.39.Fe,13.75.Jz,14.20.Jn 


\section{INTRODUCTION}

There are some different views about the structure of $\Lambda(1405)$, and its structure has been challenging the standard view of baryons made of three quarks for decades. Some theorists think that the $\Lambda(1405)$ could be a kind of molecular state arising from the interaction of the $\pi \Sigma$ and $\bar{K} N$ channels [1 $[3]$. Furthermore, the number of poles on the complex energy plane is also a puzzle for the $\Lambda(1405)$ resonance. Some people predict one pole corresponds to the $\Lambda(1405)$ resonance [1, 4, 5], while the calculation results in the unitary coupled-channel approximation show there are two poles in the $1400 \mathrm{MeV}$ region [6 10]. Two $\Lambda(1405)$ states are claimed for the first time in Ref. [6], which are explained as combinations of a single state and a octet state when the $S U(3)$ symmetry breaks up [7]. It is reported that only one resonance state is observed around $1400 \mathrm{MeV}\left[11\right.$ 14]. However, in a modern $K^{+\Sigma} \pi$ photoproduction experiment made by CLAS Collaboration, some signature effect for a twopole picture of the isospin $I=0 \Lambda(1405)$ has been found[15].

In the past years, the energy shift and width of the 1s state of the Kaonic hydrogen are measured precisely in the SIDDHARTA experiment at $D A \Phi N E[16$, which provide a constraint on the determination of parameters in the calculation of unitary coupled-channel approximation. The new experimental data have stimulated the theoretical study on the

Kaon-nucleon interaction and the properties of the $\Lambda(1405)$ particle [17 20], where the BetheSalpeter equation is solved in the unitary coupled-channel approximation, and the twopole picture of the $\Lambda(1405)$ particle is stressed. The direct comparison of the most recent approaches is made in Ref. [21]. However, a loop function of the meson and the baryon in the on-shell factorization is used when the Bethe-Salpeter equation is solved, and some important elements might be eliminated in this approximation, which would result in the uncertainty of the calculation. In this article, we calculate the loop function in a dimensional regularization scheme, and then solve the Bethe-Salpeter equation in the unitary coupledchannel approximation.

This manuscript is organized as follows. In Section [II, the framework of unitary coupledchannel approximation is discussed, especially, the loop function of the pseudoscalar meson and the baryon octet is obtained in the dimensional regularization. In Section III, the parameters in the unitary coupled-channel approximation are determined according to the experimental data at the $K^{-} p$ threshold. In Sections [V] and [V] The cases with isospin $I=0$ and $I=1$ are calculated, respectively. Some discussions and a conclusion are given in Section VI.

\section{FRAMEWORK}

The lowest order meson-baryon chiral Lagrangian is given as $[22,24]$

$$
L=\left\langle\bar{B} i \gamma^{\mu} \frac{1}{4 f^{2}}\left[\left(\Phi \partial_{\mu} \Phi-\partial_{\mu} \Phi \Phi\right) B-B\left(\Phi \partial_{\mu} \Phi-\partial_{\mu} \Phi \Phi\right)\right]\right\rangle,
$$


where the symbol $\langle\ldots\rangle$ denotes the trace of matrices in the $S U(3)$ space. The matrices of the pseudoscalar meson and the baryon octet are given as follows

$$
\Phi=\left(\begin{array}{ccc}
\frac{1}{\sqrt{2}} \pi^{0}+\frac{1}{\sqrt{6}} \eta & \pi^{+} & K^{+} \\
\pi^{-} & -\frac{1}{\sqrt{2}} \pi^{0}+\frac{1}{\sqrt{6}} \eta & K^{0} \\
K^{-} & \bar{K}^{0} & -\frac{2}{\sqrt{6}} \eta
\end{array}\right)
$$

and

$$
B=\left(\begin{array}{ccc}
\frac{1}{\sqrt{2}} \Sigma^{0}+\frac{1}{\sqrt{6}} \Lambda & \Sigma^{+} & p \\
\Sigma^{-} & -\frac{1}{\sqrt{2}} \Sigma^{0}+\frac{1}{\sqrt{6}} \Lambda & n \\
\Xi^{-} & \Xi^{0} & -\frac{2}{\sqrt{6}} \Lambda
\end{array}\right) .
$$

Ten coupled channels are considered in the case of pseudoscalar meson-baryon octet scattering processes at the low energy region, which are $K^{-} p, \bar{K}^{0} n, \pi^{0} \Lambda, \pi^{0} \Sigma^{0}, \pi^{+} \Sigma^{-}, \pi^{-} \Sigma^{+}$, $\eta \Lambda, \eta \Sigma^{0}, K^{+} \Xi^{-}$and $K^{0} \Xi^{0}[7]$.

The potential of the baryon octet and the pseudoscalar meson can be obtained from the lowest order meson-baryon chiral Lagrangian in Eq. (1), and can be written as

$$
V_{i j}=-C_{i j} \frac{1}{4 f^{2}} \bar{U}\left(p_{2}\right) \gamma_{\mu} U\left(p_{1}\right)\left(k_{1}^{\mu}+k_{2}^{\mu}\right)
$$

where $p_{1}, p_{2}\left(k_{1}, k_{2}\right)$ are the initial and final momenta of baryons (mesons), respectively. In the case of low energies, the three-momenta of baryons and mesons can be neglected, and thus only the $\gamma^{0}$ component is relevant, i.e.,

$$
\bar{U}\left(p_{2}\right) \gamma_{\mu} U\left(p_{1}\right) \approx\left(\frac{M_{i}+E}{2 M_{i}}\right)^{\frac{1}{2}}\left(\frac{M_{j}+E^{\prime}}{2 M_{j}}\right)^{\frac{1}{2}}
$$

where $M_{i}$ and $M_{j}$ denote the initial and final baryon masses, respectively, while $E$ and $E^{\prime}$ stand for the initial and final baryon energies in the center of mass frame, respectively. The spin orientation of the initial baryon is the same as that of the final baryon. Moreover,

$$
k_{1}^{0}+k_{2}^{0} \approx 2 \sqrt{s}-M_{i}-M_{j},
$$

with $\sqrt{s}$ the total energy of the system in the center of mass frame.

Therefore, if and only if the external particles are all on-shell, the potential in Eq. (4) takes a simple form as follow [7]:

$$
V_{i j}=-C_{i j} \frac{1}{4 f^{2}}\left(2 \sqrt{s}-M_{i}-M_{j}\right)\left(\frac{M_{i}+E}{2 M_{i}}\right)^{\frac{1}{2}}\left(\frac{M_{j}+E^{\prime}}{2 M_{j}}\right)^{\frac{1}{2}},
$$

where the coefficient $C_{i j}$ is shown in Table $\mathbf{V}$, and the decay constant $f=1.123 f_{\pi}$ [9] with the pion decay constant $f_{\pi}=92.4 \mathrm{MeV}$. 
The scattering amplitude can be constructed by solving the Bethe-Salpeter equation

$$
\begin{aligned}
T\left(p_{1}, k_{2} ; p_{2}, k_{2}\right) & =V\left(p_{1}, k_{2} ; p_{2}, k_{2}\right) \\
& +i \int \frac{d^{4} q}{(2 \pi)^{4}} V\left(p_{1}, k_{1} ; q, p_{1}+k_{1}-q\right) S(q) \Delta\left(p_{1}+k_{1}-q\right) T\left(q, p_{1}+k_{1}-q ; p_{2}, k_{2}\right) \\
& =V\left(p_{1}, k_{2} ; p_{2}, k_{2}\right) \\
& +i \int \frac{d^{4} q}{(2 \pi)^{4}} V\left(p_{1}, k_{1} ; q, p_{1}+k_{1}-q\right) S(q) \Delta\left(p_{1}+k_{1}-q\right) V\left(q, p_{1}+k_{1}-q ; p_{2}, k_{2}\right) \\
& +\ldots,
\end{aligned}
$$

where the propagators of the intermediate baryon and meson can be written as $i S(q)=$ $i /\left(\not 1+M_{l}+i \varepsilon\right)$ and $i \Delta\left(p_{1}+k_{1}-q\right)=i /\left[\left(p_{1}+k_{1}-q\right)^{2}-m_{l}^{2}+i \epsilon\right]$, respectively [25]. If the potential $V\left(p_{1}, k_{1} ; q, p_{1}+k_{1}-q\right)$ in Eq. (8) is divided into a on-shell part and an off-shell part, the off-shell part of the potential $V\left(p_{1}, k_{1} ; q, p_{1}+k_{1}-q\right)$ would be proportional to the on-shall part, and can be absorbed into the on-shell part of the potential if a suitable renormalization of coupling constants is preformed. Therefore, only the on-shell part of the potential of the baryon and the meson is necessary to be taken into account when the Bethe-Salpeter equation is solved. Thus the Bethe-Salpeter equation in Eq. (8) is simplified as

$$
T=V+V G T
$$

or

$$
T=[1-V G]^{-1} V
$$

where the loop function of a baryon and a meson $G$ is a diagonal matrix, i.e., $G_{l n}=G_{l} \delta_{l n}$, and the diagonal element $G_{l}$ can be written as

$$
G_{l}=i \int \frac{d^{4} q}{(2 \pi)^{4}} \frac{\not 1+M_{l}}{q^{2}-M_{l}^{2}+i \epsilon} \frac{1}{(P-q)^{2}-m_{l}^{2}+i \epsilon}
$$

with $P=p_{1}+k_{1}$ the total momentum of the system, $m_{l}$ the meson mass, and $M_{l}$ the baryon mass, respectively. More detailed discussion on how to transform the Bethe-Salpeter equation from an integral form to an algebra form can be found in Refs. [25, 26].

In Ref. [26], the loop function $G$ in Eq. (11) is calculated numerically by setting the maximum three-momentum $q_{\max }$, which is called the momenum cut-off method. However, in Ref. [6], a dimensional regularization form of the loop function $G$ is deduced with the on-shell approximation

$$
\not 1+M_{l}=2 M_{l},
$$

which is valid only when it is applied on the Dirac spinor $U(q)$. 
In the on-shell factorization approximation, the loop function is denoted as

$$
\begin{aligned}
& G_{l}^{\prime}(s)= i \int \frac{d^{d} q}{(2 \pi)^{4}} \frac{2 M_{l}}{q^{2}-M_{l}^{2}+i \epsilon} \frac{1}{(P-q)^{2}-m_{l}^{2}+i \epsilon} \\
&=\frac{2 M_{l}}{16 \pi^{2}}\left\{a_{l}+\ln \frac{M_{l}^{2}}{\mu^{2}}+\frac{m_{l}^{2}-M_{l}^{2}+s}{2 s} \ln \frac{m_{l}^{2}}{M_{l}^{2}}+\right. \\
& \quad+\frac{\bar{q}_{l}}{\sqrt{s}}\left[\ln \left(s-\left(M_{l}^{2}-m_{l}^{2}\right)+2 \bar{q}_{l} \sqrt{s}\right)+\ln \left(s+\left(M_{l}^{2}-m_{l}^{2}\right)+2 \bar{q}_{l} \sqrt{s}\right)\right. \\
&\left.\left.\quad-\ln \left(-s+\left(M_{l}^{2}-m_{l}^{2}\right)+2 \bar{q}_{l} \sqrt{s}\right)-\ln \left(-s-\left(M_{l}^{2}-m_{l}^{2}\right)+2 \bar{q}_{l} \sqrt{s}\right)\right]\right\}
\end{aligned}
$$

with $\mu=630 \mathrm{MeV}$ the scale of dimensional regularization and the symbol $a_{l}$ the subtraction constant.

In Eq. (13), $\bar{q}_{l}$ denotes the three-momentum of the meson or the baryon in the center of mass frame and is given by

$$
\bar{q}_{l}=\frac{\lambda^{1 / 2}\left(s, m_{l}^{2}, M_{l}^{2}\right)}{2 \sqrt{s}}=\frac{\sqrt{s-\left(M_{l}+m_{l}\right)^{2}} \sqrt{s-\left(M_{l}-m_{l}\right)^{2}}}{2 \sqrt{s}},
$$

with $\lambda$ the triangular function.

Actually, the loop function in Eq. (11) can be calculated in the dimensional regularization without the on-shell approximation taken into account. Thus the loop function takes the form of

$$
\begin{aligned}
G_{l}= & \frac{\gamma_{\mu} P^{\mu}}{32 P^{2} \pi^{2}}\left[\left(a_{l}+1\right)\left(m_{l}^{2}-M_{l}^{2}\right)+\left(m_{l}^{2} \ln \frac{m_{l}^{2}}{\mu^{2}}-M_{l}^{2} \ln \frac{M_{l}^{2}}{\mu^{2}}\right)\right] \\
& +\left(\frac{\gamma_{\mu} P^{\mu}\left[P^{2}+M_{l}^{2}-m_{l}^{2}\right]}{4 P^{2} M_{l}}+\frac{1}{2}\right) G_{l}^{\prime} .
\end{aligned}
$$

Since the total three-momentum $\vec{P}=0$ in the center of mass frame, only the $\gamma_{0} P^{0}$ parts remain in Eq. (15). The external particles in the potential of the baryon and the meson in Eq. (77) are all on-shell, so the anti-particle is not included in the intermediate states when the Bethe-Salpeter equation is solved. Thus $\gamma_{0} P^{0}$ can be treated as the total energy of the system $P^{0}=\sqrt{s}$ in Eq. (15). Therefore, the loop function in Eq. (15) is simplified as

$$
\begin{aligned}
G_{l}= & \frac{\sqrt{s}}{32 \pi^{2} s}\left[\left(a_{l}+1\right)\left(m_{l}^{2}-M_{l}^{2}\right)+\left(m_{l}^{2} \ln \frac{m_{l}^{2}}{\mu^{2}}-M_{l}^{2} \ln \frac{M_{l}^{2}}{\mu^{2}}\right)\right] \\
& +\left(\frac{s+M_{l}^{2}-m_{l}^{2}}{4 M_{l} \sqrt{s}}+\frac{1}{2}\right) G_{l}^{\prime}
\end{aligned}
$$

Apparently, some off-shell corrections have been included in the revised form of the loop function in Eq. (16), which can be regarded as a kind of relativistic kinetic effect of the loop function.

Assuming the amplitudes near the pole to behave as

$$
T_{i j}=\frac{g_{i} g_{j}}{z-z_{R}}
$$


with $z_{R}$ the position of the pole on the complex $\sqrt{s}$ plane, and $j$ and $i$ being the initial and final channels, respectively, we can obtain the size of the coupling constants $g_{i}$ by evaluating the residues of the diagonal elements $T_{i i}$. When the strongest coupled channel is determined, the coupling constants to the other channels, $g_{j}$, can be evaluated with the residues of the non-diagonal elements $T_{i j}$ and the largest coupling constant $g_{i}$ by using Eq. (17).

\section{EXPERIMENTAL DATA AND PARAMETER FITS}

The energy shift and the width of the 1s state of the Kaonic hydrogen measured by the SIDDHARTA Collaboration are given as

$$
\Delta E=283 \pm 36 \pm 6 e V
$$

and

$$
\Gamma=541 \pm 89 \pm 22 e V
$$

respectively[16]. These results would supply a constraint to the parameter fit when the Bethe-Salpeter equation is solved.

In order to fit the experimental data at the $K^{-} p$ threshold with the same formula as in Ref. [17], the potential of the baryon octet and the pseudoscalar meson in Eq. (77) has to be multiplied by a factor of $\sqrt{M_{i} M_{j}}$, where $M_{i}$ and $M_{j}$ denote the masses of the initial and final baryons, respectively, i.e.,

$$
\tilde{V}_{i j}=V_{i j} \sqrt{M_{i} M_{j}}
$$

In the meantime, the loop function in Eq. (16) is divided by the mass of the intermediate baryon $M_{l}$, i.e.,

$$
\tilde{G}_{l}=G_{l} / M_{l}
$$

Since the pole appears when the determinant $|1-V G|=0$, the modification in Eqs. (20) and (21) will not affect the pole position on the complex $\sqrt{s}$ plane significantly.

The forward scattering amplitude $f_{i j}$ is related to the T-matrix,

$$
f_{i j}(\sqrt{s})=\frac{1}{8 \pi \sqrt{s}} \tilde{T}_{i j}(\sqrt{s})
$$

with $\tilde{T}=[1-\tilde{V} \tilde{G}]^{-1} \tilde{V}$, and the $K^{-} p$ scattering length can be defined by the $K^{-} p$ elastic scattering amplitude at threshold,

$$
a\left(K^{-} p\right)=f_{11}\left(\sqrt{s}=M_{K^{-}}+m_{p}\right)
$$

which is a complex number when the inelastic channels are taken into account.

The energy shift and width of the 1 s state of the Kaonic hydrogen are related to the $K^{-} p$ scattering length, which can be written as 27]

$$
\Delta E-i \frac{1}{2} \Gamma=-2 \alpha^{3} \mu_{\gamma}^{2} a\left(K^{-} p\right)\left[1+2 \alpha \mu_{\gamma}(1-\ln \alpha) a\left(K^{-} p\right)\right],
$$


with $\alpha$ the fine structure constant and $\mu_{\gamma}=\frac{m_{K^{-}} M_{p}}{m_{K^{-}}+M_{p}}$ the $K^{-} p$ reduced mass.

Moreover, the branching ratios at the $K^{-} p$ threshold defined as

$$
\begin{gathered}
\gamma=\frac{\Gamma\left(K^{-} p \rightarrow \pi^{+} \Sigma^{-}\right)}{\Gamma\left(K^{-} p \rightarrow \pi^{-} \Sigma^{+}\right)}=\frac{\sigma_{51}}{\sigma_{61}} \\
R n=\frac{\Gamma\left(K^{-} p \rightarrow \pi^{0} \Lambda\right)}{\Gamma\left(K^{-} p \rightarrow \text { neutral states }\right)}=\frac{\sigma_{31}}{\sigma_{31}+\sigma_{41}},
\end{gathered}
$$

and

$$
R c=\frac{\Gamma\left(K^{-} p \rightarrow \pi^{+} \Sigma^{-}, \pi^{-} \Sigma^{+}\right)}{\Gamma\left(K^{-} p \rightarrow \text { all inelastic channels }\right)}=\frac{\sigma_{51}+\sigma_{61}}{\sigma_{51}+\sigma_{61}+\sigma_{31}+\sigma_{41}},
$$

respectively. All partial cross sections $\sigma_{i j}$ are calculated at the $K^{-} p$ threshold.

$$
\sigma_{i j}=\frac{\bar{q}_{i}}{\bar{q}_{j}} \frac{\left|\tilde{T}_{i j}\right|^{2}}{16 \pi s}
$$

where $\bar{q}_{j}$ and $\bar{q}_{i}$ are the three-momentum of the initial and final states in the center of mass frame, respectively.

The values of three branching ratios are taken from Ref. [28, 29], i.e.,

$$
\gamma=2.36 \pm 0.04, \quad R_{n}=0.189 \pm 0.015, \quad R_{c}=0.664 \pm 0.011
$$

The subtraction constants $a_{l}$ in Eq. (16) for different channels can be determined according to experimental data at the $K^{-} p$ threshold, which are labeled with Off - shell in Table I. Moreover, the corresponding subtraction constants in the original on-shell factorization approximation in Eq. (13) are also listed in Table I, which is labeled with On-shell [9].

\begin{tabular}{ccccccc}
\hline \hline$a_{l}$ & $\bar{K} N$ & $\pi \Lambda$ & $\pi \Sigma$ & $\eta \Lambda$ & $\eta \Sigma$ & $K \Xi$ \\
\hline On-shell & -1.84 & -1.83 & -2.0 & -2.25 & -2.38 & -2.67 \\
Off-shell & -1.1 & -1.6 & -1.9 & -2.7 & -2.6 & -2.8 \\
\hline \hline
\end{tabular}

TABLE I: The subtraction constants used in the calculation with $\mu=630 \mathrm{MeV}$. The label $O f f-$ shell denotes the values for the loop function in Eq. (16), where some off-shell corrections are taken into account, while the label $\mathrm{On}$ - shell stands for those original values in the on-shell factorization approximation in Eq. (13) [9].

The corresponding values of the energy shift $\Delta E$ and width $\Gamma$ of the 1 s state of the Kaonic hydrogen, the braching ratios $R_{n}$ and $R_{c}$ defined in Eqs. (26) and (27) reproduced with the loop function in Eq. (16) are listed in Table II, and the subtraction constants labeled with Off - shell are used. These values are also calculated with the $O n-$ shell subtraction constants in the on-shell factorization approximation. The results show that the Off $f$ shell subtraction constants fitted with the experiment data at the $K^{-} p$ threshold are reasonable. 


\begin{tabular}{ccccc}
\hline \hline & $\Delta E[\mathrm{eV}]$ & $\Gamma \quad[\mathrm{eV}]$ & $R n$ & $R c$ \\
\hline Experimental data & $283 \pm 36 \pm 6$ & $541 \pm 89 \pm 22$ & $0.189 \pm 0.015$ & $0.664 \pm 0.011$ \\
On-shell & -180.11 & 444.14 & 0.28 & 0.61 \\
Off-shell & 283.13 & 541.06 & 0.3 & 0.61 \\
\hline \hline
\end{tabular}

TABLE II: The energy shift $\Delta E$ and width $\Gamma$ of the 1 s state of the Kaonic hydrogen, the braching ratios $R_{n}$ and $R_{c}$ defined in Eqs. (26) and (27) calculated with different subtraction constant sets. The meanings of labels $O n-$ shell and $O f f-$ shell are the same as that in Table I.

Since the meson-baryon amplitude $T_{i j}$ is calculated in the isospin sectors, the subtraction constant $a_{K N}$ is supposed to take the same value both in the $K^{-} p$ channel and in the $\bar{K}^{0} n$ channel. Thus the branching ratio $\gamma$ is always one in our calculation.

\section{THE ISOSPIN $I=0$ SECTOR}

We shall discuss the scattering amplitude in the isospin states, and thus we must use the average mass for the $\pi\left(\pi^{+}, \pi^{0}, \pi^{-}\right), K\left(K^{+}, K^{0}\right), \bar{K}\left(\bar{K}^{0}, K^{-}\right), N(p, n), \Sigma\left(\Sigma^{+}, \Sigma^{0}, \Sigma^{-}\right)$ and $\Xi\left(\Xi^{0}, \Xi^{-}\right)$particles. There are four coupled states with isospin $I=0$ and strangeness $S=-1$, which are $\bar{K} N, \pi \Sigma, \eta \Lambda$ and $K \Xi$.

The phase conventions $\left|\pi^{+}\right\rangle=-|1,1\rangle,\left|K^{-}\right\rangle=-\left|\frac{1}{2},-\frac{1}{2}\right\rangle,\left|\Sigma^{+}\right\rangle=-|1,1\rangle$ and $\left|\Xi^{-}\right\rangle=$ $-\left|\frac{1}{2},-\frac{1}{2}\right\rangle$ are used for the isospin states, which are consistent with the structure of the $\Phi$ and $B$ matrices, and then the isospin state with $I=0$ can be written as

$$
\begin{aligned}
|\bar{K} N, I=0\rangle & =\frac{1}{\sqrt{2}}\left(\bar{K}^{0} n+K^{-} p\right), \\
|\pi \Sigma, I=0\rangle & =-\frac{1}{\sqrt{3}}\left(\pi^{+} \Sigma^{-}+\pi^{0} \Sigma^{0}+\pi^{-} \Sigma^{+}\right), \\
|K \Xi, I=0\rangle & =-\frac{1}{\sqrt{2}}\left(K^{0} \Xi^{0}+K^{+} \Xi^{-}\right) .
\end{aligned}
$$

The correspond coefficients $C_{i j}$ for the isospin states with $I=0$ are listed in Table VI. With these coefficients, the amplitudes $T$ with isospin $I=0$ can be obtained by solving the Bethe-Salpeter equation in Eq. (10).

The squared amplitude $|T|^{2}$ in the $\pi \Sigma$ channel with isospin $I=0$ on the complex $\sqrt{s}$ plane is shown in Fig. 1. Some poles are generated dynamically when the Bethe-Salpeter equation is solved in the unitary coupled-channel approximation. In Fig. 1, the poles generated dynamically with the loop function in Eq. (16) are labeled with $N E W$, while the label $P R E$ denotes the poles generated with the loop function in the on-shell factorization in Eq. (13). In the energy region near $1400 \mathrm{MeV}$, it can be seen that there is only one pole generated dynamically in the isospin $I=0$ sector, which locates at $1383+81 i \mathrm{MeV}$ on the complex $\sqrt{s}$ plane. This pole is higher than the $\pi \Sigma$ threshold and lies in the second Riemann sheet, and thus it can be regarded as a counterpart of the $\Lambda(1405)$ particle in the data of 
Particle Data Group(PDG) [30]. Actually, there is another peak generated dynamically, which is at $1435+2 i \mathrm{MeV}$ on the complex $\sqrt{s}$ plane. However, it is too low to be detected in Fig. 1. Apparently, when the off-shell correction of the loop function in the Bethe-Salpeter equation is taken into account, the peak near the $\bar{K} N$ threshold is suppressed strongly, and only one pole is detected in the region of $1400 \mathrm{MeV}$. The resonance at $1383+81 i \mathrm{MeV}$ couples strongly to the $\pi \Sigma$ channel. In the $\bar{K} N, \eta \Lambda$ and $K \Xi$ channels, the cases are similar to that in the $\pi \Sigma$ channel, and only one pole can be found clearly. Furthermore, there is another pole at $1653+12 i \mathrm{MeV}$ generated dynamically on the complex $\sqrt{s}$ plane, which is lower than the $\eta \Lambda$ threshold, and lies in the third Riemann sheet. The pole at the position of $\sqrt{S}=1653+12 i \mathrm{MeV}$ can be regarded as a candidate of the $\Lambda(1670) 1 / 2^{-}$resonance, which couples strongly to the $K \Xi$ channel.

The coupling constants of them to different meson-baryon states are listed in Table. III, where the label $N e w$ denotes the results with the loop function in Eq. (16), while the label $P R E$ means the results calculated with the loop function in the on-shell factorization, as in Eq. (13).

\section{THE ISOSPIN $I=1$ SECTOR}

In the isospin $I=1$ sector, we have five coupled states, $\bar{K} N, \pi \Sigma, \pi \Lambda, \eta \Sigma$ and $K \Xi$. The isospin state with $I=1$ can be written as

$$
\begin{aligned}
|\bar{K} N, I=1\rangle & =\frac{1}{\sqrt{2}}\left(\bar{K}^{0} n-K^{-} p\right), \\
|\pi \Sigma, I=1\rangle & =\frac{1}{\sqrt{2}}\left(\pi^{-} \Sigma^{+}-\pi^{+} \Sigma^{-}\right), \\
|K \Xi, I=1\rangle & =\frac{1}{\sqrt{2}}\left(K^{0} \Xi^{0}-K^{+} \Xi^{-}\right) .
\end{aligned}
$$

The coefficients $C_{i j}$ for the isospin states with $I=1$ can be constructed by using Eq. (31) and Table $\mathrm{V}$, which are given in Table VII.

There is only one peak of squared amplitudes $|T|^{2}$ detected at $1570+i 244 \mathrm{MeV}$ on the complex $\sqrt{s}$ plane, which is lower than $\eta \Sigma$ threshold and lies in the fourth Riemann sheet. The resonance at $1570+i 244 \mathrm{MeV}$ is similar to the $\Sigma(1580) 3 / 2$ - state in the PDG data. Nevertheless, in the S-wave approximation, the total angular momentum of the resonance at $1570+i 244 \mathrm{MeV}$ is $J=1 / 2$, and its parity is negative, furthermore, the decay width is too large, so it can not be $\Sigma(1580) 3 / 2$ - apparently. The resonance at $1570+i 244 \mathrm{MeV}$ is more possible to correspond to the $\Sigma(1620) 1 / 2$ - state in the $P D G$ data although its mass is lower about $50 \mathrm{MeV}$ than the latter. From Table IV, we can know that this resonance couples to the $K \Xi$ channel strongly.

The squared amplitude $|T|^{2}$ in the $\pi \Sigma$ channel with strangeness $S=-1$ and isospin $I=1$ as a function of the total energy $\sqrt{s}$ in the center of mass frame is depicted in Fig. 2, where the label PRE denotes the results calculated with the loop function in the on-shell 


\begin{tabular}{|c|c|c|c|c|}
\hline & \multicolumn{2}{|c|}{ PRE } & \multicolumn{2}{|c|}{ NEW } \\
\hline$\overline{z_{R}}$ & \multicolumn{2}{|c|}{$1390+66 i$} & \multicolumn{2}{|c|}{$1383+81 i$} \\
\hline$(I=0)$ & $g_{i}$ & $\left|g_{i}\right|$ & $g_{i}$ & $\left|g_{i}\right|$ \\
\hline$\pi \Sigma$ & $-2.5-1.5 i$ & 2.9 & $-2.1-1.5 i$ & 2.5 \\
\hline $\bar{K} N$ & $1.2+1.7 i$ & 2.1 & $0.8+0.8 i$ & 1.1 \\
\hline$\eta \Lambda$ & $0.0+0.8 i$ & 0.8 & $-0.1+0.2 i$ & 0.24 \\
\hline \multirow[t]{2}{*}{$K \Xi$} & $-0.5-0.4 i$ & 0.6 & $-0.4-0.5 i$ & 0.6 \\
\hline & \multicolumn{2}{|c|}{ PRE } & \multicolumn{2}{|c|}{ NEW } \\
\hline$z_{R}$ & \multicolumn{2}{|c|}{$1426+16 i$} & \multicolumn{2}{|c|}{-} \\
\hline$(I=0)$ & $g_{i}$ & $\left|g_{i}\right|$ & $g_{i}$ & $\left|g_{i}\right|$ \\
\hline$\pi \Sigma$ & $0.4-1.4 i$ & 1.5 & - & - \\
\hline $\bar{K} N$ & $-2.5+0.9 i$ & 2.7 & - & - \\
\hline$\eta \Lambda$ & $-1.4+0.2 i$ & 1.4 & - & - \\
\hline \multirow[t]{2}{*}{$K \Xi$} & $0.1-0.3 i$ & 0.4 & - & - \\
\hline & \multicolumn{2}{|c|}{ PRE } & \multicolumn{2}{|c|}{ NEW } \\
\hline$\overline{z_{R}}$ & \multicolumn{2}{|c|}{$1680+20 i$} & \multicolumn{2}{|c|}{$1653+12 i$} \\
\hline$(I=0)$ & $g_{i}$ & $\left|g_{i}\right|^{2}$ & $g_{i}$ & $\left|g_{i}\right|^{2}$ \\
\hline$\pi \Sigma$ & $-0.0-0.3 i$ & 0.3 & $-0.1-0.3 i$ & 0.3 \\
\hline $\bar{K} N$ & $0.3+0.7 i$ & 0.8 & $-0.0+0.7 i$ & 0.7 \\
\hline$\eta \Lambda$ & $-1.1-0.1 i$ & 1.1 & $-1.1+0.2 i$ & 1.1 \\
\hline$K \Xi$ & $3.4+0.1 i$ & 3.5 & $3.0-0.1 i$ & 3.0 \\
\hline
\end{tabular}

TABLE III: Coupling constants to meson-baryon states in the isospin $I=0$ sector. The label $N E W$ denotes the case calculated from the loop function in Eq. (16), while the label PRE stands for the case of the loop function in the on-shell factorization approximation in Eq. (13).

factorization, and the label $N E W$ means those of the loop function in Eq. (16). The pole positions are different in these two schemes.

\section{DISCUSSION AND CONCLUSION}

In this article, the formula of the loop function in the Bethe-Salpeter equation is deduced in the dimensional regularization scheme. Comparing with the loop function in the on-shell factorization approximation, the relativistic kinetic effect and off-shell corrections are taken into account in the revised scheme.

The interaction between the pseudoscalar meson and the baryon is studied in the strangeness $S=-1$ sector. According to the experimental data at the $K^{-} p$ threshold, the subtraction constants of the loop function in the Bethe-Salpeter equation are determined, and some resonances are generated dynamically in the unitary coupled-channel approxima- 


\begin{tabular}{ccccc}
\hline \hline \multicolumn{2}{c}{ PRE } & \multicolumn{2}{c}{ NEW } \\
\hline$z_{R}$ & \multicolumn{1}{c}{$1579+264 i$} & & $1570+244 i$ & \\
$(I=1)$ & $g_{i}$ & $\left|g_{i}\right|^{2}$ & $g_{i}$ & $\left|g_{i}\right|^{2}$ \\
\hline$\pi \Lambda$ & $1.4+1.5 i$ & 2.0 & $1.3+1.4 i$ & 1.9 \\
$\pi \Sigma$ & $-2.2-1.5 i$ & 2.7 & $-2.2-1.2 i$ & 2.5 \\
$\bar{K} N$ & $-1.1-1.1 i$ & 1.6 & $-1.0-1.2 i$ & 1.6 \\
$\eta \Sigma$ & $1.2+1.4 i$ & 1.9 & $1.1+1.3 i$ & 1.7 \\
$K \Xi$ & $-2.5-2.4 i$ & 3.5 & $-2.4-2.1 i$ & 3.2 \\
\hline \hline
\end{tabular}

TABLE IV: Same as Table III but for the isospin $I=1$ sector.

tion. When the off-shell correction of the loop function is taken into account, the squared amplitude is suppressed strongly, and only one resonance peak is detected in the $1400 \mathrm{MeV}$ region, which is above the $\pi \Sigma$ threshold and is assumed to be associated to the $\Lambda(1405)$ particle. Moreover, the coupling constants of the resonance to meson-baryon states are similar to those in Ref. [7] correspondingly, and the resonance in the $1400 \mathrm{MeV}$ region couples strongly to the $\pi \Sigma$ channel.

\section{Acknowledgments}

We would like to thank Han-Qing Zheng, Eulogio Oset and En Wang for useful discussions.

\section{Appendix 1: A loop function in the dimensional regularization scheme}

In the dimensional regularization scheme, the loop function in the Bethe-Salpeter equation can be deduced according to the Passarino-Veltman procedure 31],

$$
G_{l}(s)=i \int \frac{d^{4} q}{(2 \pi)^{4}} \frac{\not l+M_{l}}{q^{2}-M_{l}^{2}+i \epsilon} \frac{1}{(P-q)^{2}-m_{l}^{2}+i \epsilon}=-P A(s)+\frac{1}{2} G^{\prime}(s),
$$

where

$$
\begin{aligned}
& G^{\prime}(s)= i \int \frac{d^{4} q}{(2 \pi)^{4}} \frac{2 M_{l}}{q^{2}-M_{l}^{2}+i \epsilon} \frac{1}{(P-q)^{2}-m_{l}^{2}+i \epsilon} \\
&=\frac{2 M_{l}}{16 \pi^{2}}\left\{a_{l}+\ln \frac{M_{l}^{2}}{\mu^{2}}+\frac{m_{l}^{2}-M_{l}^{2}+s}{2 s} \ln \frac{m_{l}^{2}}{M_{l}^{2}}+\right. \\
& \quad+\frac{\bar{q}_{l}}{\sqrt{s}}\left[\ln \left(s-\left(M_{l}^{2}-m_{l}^{2}\right)+2 \bar{q}_{l} \sqrt{s}\right)+\ln \left(s+\left(M_{l}^{2}-m_{l}^{2}\right)+2 \bar{q}_{l} \sqrt{s}\right)\right. \\
&\left.\left.\quad-\ln \left(-s+\left(M_{l}^{2}-m_{l}^{2}\right)+2 \bar{q}_{l} \sqrt{s}\right)-\ln \left(-s-\left(M_{l}^{2}-m_{l}^{2}\right)+2 \bar{q}_{l} \sqrt{s}\right)\right]\right\},(
\end{aligned}
$$


and

$$
\begin{gathered}
P^{\mu} A(s)=-i \int \frac{d^{4} q}{(2 \pi)^{4}} \frac{q^{\mu}}{q^{2}-M_{l}^{2}+i \epsilon} \frac{1}{(P-q)^{2}-m_{l}^{2}+i \epsilon} . \\
\text { Since }-2 P \cdot q=\left[(P-q)^{2}-m_{l}^{2}\right]-\left[q^{2}-M_{l}^{2}\right]+m_{l}^{2}-M_{l}^{2}-P^{2} \\
-2 P^{2} A(s)=-i \int \frac{d^{4} q}{(2 \pi)^{4}} \frac{-2 P \cdot q}{q^{2}-M_{l}^{2}+i \epsilon} \frac{1}{(P-q)^{2}-m_{l}^{2}+i \epsilon} \\
=-i \int \frac{d^{4} q}{(2 \pi)^{4}} \frac{\left[(P-q)^{2}-m_{l}^{2}\right]-\left[q^{2}-M_{l}^{2}\right]+m_{l}^{2}-M_{l}^{2}-P^{2}}{\left[q^{2}-M_{l}^{2}+i \epsilon\right]\left[(P-q)^{2}-m_{l}^{2}+i \epsilon\right]} \\
=-i \int \frac{d^{4} q}{(2 \pi)^{4}} \frac{1}{q^{2}-M_{l}^{2}+i \epsilon}-i \int \frac{d^{4} q}{(2 \pi)^{4}} \frac{-1}{(P-q)^{2}-m_{l}^{2}+i \epsilon} \\
-i \int \frac{d^{4} q}{(2 \pi)^{4}} \frac{m_{l}^{2}-M_{l}^{2}-P^{2}}{\left[q^{2}-M_{l}^{2}+i \epsilon\right]\left[(P-q)^{2}-m_{l}^{2}+i \epsilon\right]} \\
=-\frac{M_{l}^{2}}{16 \pi^{2}}\left(1+a_{l}+\ln \left(\frac{M_{l}^{2}}{\mu}\right)\right)+\frac{m_{l}^{2}}{16 \pi^{2}}\left(1+a_{l}+\ln \left(\frac{m_{l}^{2}}{\mu}\right)\right)-\frac{\left(P^{2}+M_{l}^{2}-m_{l}^{2}\right)}{2 M_{l}}\left(-G^{\prime}(s)\right) .
\end{gathered}
$$

Thus the formula of $A(s)$ can be written as

$A(s)=\frac{1}{-2 P^{2}}\left\{-\frac{M_{l}^{2}}{16 \pi^{2}}\left(1+a_{l}+\ln \left(\frac{M_{l}^{2}}{\mu}\right)\right)+\frac{m_{l}^{2}}{16 \pi^{2}}\left(1+a_{l}+\ln \left(\frac{m_{l}^{2}}{\mu}\right)\right)-\frac{\left(P^{2}+M_{l}^{2}-m_{l}^{2}\right)}{2 M_{l}}\left(-G^{\prime}(s)\right)\right\}$

with $P^{2}=s$.

By replacing the $G^{\prime}(s)$ and $A(s)$ in Eq. (A1) with Eqs. (A2) and (A3), respectively, the formula of the loop function in Eq. (15) is obtained. 
Appendix 2: The coefficient $C_{i j}$ in the pseudoscalar meson-baryon octet interaction in the $S=-1$ channel

\begin{tabular}{|c|c|c|c|c|c|c|c|c|c|c|}
\hline & $K^{-} p$ & $\bar{K}^{0} n$ & $\pi^{0} \Lambda$ & $\overline{\pi^{0} \Sigma^{0}}$ & $\eta \Lambda$ & $\eta \Sigma^{0}$ & $\pi^{+} \Sigma^{-}$ & $\pi^{-} \Sigma^{+}$ & $K^{+} \Xi^{-}$ & $K^{0} \Xi^{0}$ \\
\hline$K^{-} p$ & 2 & 1 & $\frac{\sqrt{3}}{2}$ & $\frac{1}{2}$ & $\frac{3}{2}$ & $\frac{\sqrt{3}}{2}$ & 0 & 1 & 0 & 0 \\
\hline $\bar{K}^{0} n$ & & 2 & $-\frac{\sqrt{3}}{2}$ & $\frac{1}{2}$ & $\frac{3}{2}$ & $-\frac{\sqrt{3}}{2}$ & 1 & 0 & 0 & 0 \\
\hline$\pi^{0} \Lambda$ & & & 0 & 0 & 0 & 0 & 0 & 0 & $\frac{\sqrt{3}}{2}$ & $-\frac{\sqrt{3}}{2}$ \\
\hline$\pi^{0} \Sigma^{0}$ & & & & 0 & 0 & 0 & 2 & 2 & $\frac{1}{2}$ & $\frac{1}{2}$ \\
\hline$\eta \Lambda$ & & & & & 0 & 0 & 0 & 0 & $\frac{3}{2}$ & $\frac{3}{2}$ \\
\hline$\eta \Sigma^{0}$ & & & & & & 0 & 0 & 0 & $\frac{\sqrt{3}}{2}$ & $-\frac{\sqrt{3}}{2}$ \\
\hline$\pi^{+} \Sigma^{-}$ & & & & & & & 2 & 0 & 1 & 0 \\
\hline$\pi^{-} \Sigma^{+}$ & & & & & & & & 2 & 0 & 1 \\
\hline$K^{+} \Xi^{-}$ & & & & & & & & & 2 & 1 \\
\hline$K^{0} \Xi^{0}$ & & & & & & & & & & 2 \\
\hline
\end{tabular}

TABLE V: The coefficient $C_{i j}$ in the pseudoscalar meson-baryon octet interaction in the $S=-1$ channel, $C_{i j}=C_{j i}$.

\begin{tabular}{lllll}
\hline & $\bar{K} N$ & $\pi \Sigma$ & $\eta \Lambda$ & $K \Xi$ \\
\hline $\bar{K} N$ & 3 & $-\sqrt{\frac{3}{2}}$ & $\frac{3}{\sqrt{2}}$ & 0 \\
$\pi \Sigma$ & 4 & 0 & $\sqrt{\frac{3}{2}}$ \\
$\eta \Lambda$ & & 0 & $-\frac{3}{\sqrt{2}}$ \\
$K \Xi$ & & & 3 \\
\hline
\end{tabular}

TABLE VI: The coefficients $C_{i j}$ for the isospin states with $I=0, C_{i j}=C_{j i}$.

\begin{tabular}{llllll}
\hline & $\bar{K} N$ & $\pi \Sigma$ & $\pi \Lambda$ & $\eta \Sigma$ & $K \Xi$ \\
\hline $\bar{K} N$ & 1 & -1 & $-\sqrt{\frac{3}{2}}$ & $-\sqrt{\frac{3}{2}}$ & 0 \\
$\pi \Sigma$ & & 2 & 0 & 0 & 1 \\
$\pi \Lambda$ & & 0 & 0 & $-\sqrt{\frac{3}{2}}$ \\
$\eta \Sigma$ & & & 0 & $-\sqrt{\frac{3}{2}}$ \\
$K \Xi$ & & & & & 1 \\
\hline
\end{tabular}

TABLE VII: The coefficients $C_{i j}$ for the isospin states with $I=1, C_{i j}=C_{j i}$. 
[1] R. H. Dalitz and S. F. Tuan, Ann. Phys. (N. Y.) 10,307 (1960)

[2] R. H. Dalitz, T. C. Wong, G. Rajasekaran, Phys. Rev. 153, 1617 (1967)

[3] L. S. Kisslinger and E. M. Henley, Eur. Phys. J. A 47, 8 (2011)

[4] I. Zychor et al. Phys. Lett. B 660, 167 (2008)

[5] N. Isgur and G. Karl, Phys. Rev. D 18, 4187 (1978)

[6] J. A. Oller, U.-G Meißner, Phys. Letter. B 500, 263 (2001)

[7] D. Jido, J. A. Oller, E. Oset, A. Ramos. U.-G Meißner, Nuclear Physics A 725, 181 (2003)

[8] L. S. Geng and E. Oset, Eur. Phys. J. A 34, 405 (2007)

[9] E. Oset, A. Ramos, C. Bennhold. Phys. Lett. B 527, 99 (2002). E. Oset, A. Ramos, C. Bennhold. Erratum, Phys. Lett. B 530, 260 (2002)

[10] T. Hyodo, A. Hosaka, E. Oset, A. Ramos and M. J. Vicente Vacas, Phys. Rev. C 68, 065203 (2003)

[11] A. Cieply and J. Smejkal, Eur. Phys. J. A 43, 191 (2010)

[12] M. Hassanvand et al. Phys Rev. C 87, 055202 (2013)

[13] R. H. Dalitz and A. Deloff. J. Phys. G 17, 289 (1991)

[14] G. Alexander, G. R. Kalbfleisch, D. H. Miller et al. Phys. Rev. Lett. 8, 447 (1962)

[15] K. Moriya et al. [CLAS Collaboration], Phys. Rev. C 87, no. 3, 035206 (2013)

[16] M. Bazzi et al. [SIDDHARTA Collaboration], Phys. Lett. B 704, 113 (2011)

[17] Y. Ikeda, T. Hyodo and W. Weise, Nucl. Phys. A 881, 98 (2012)

[18] M. Mai and U. G. Meißner, Nucl. Phys. A 900, 51 (2013)

[19] Z. H. Guo and J. A. Oller, Phys. Rev. C 87, no. 3, 035202 (2013)

[20] M. Mai and U. G. Meißner, Eur. Phys. J. A 51, no. 3, 30 (2015)

[21] A. Cieply, M. Mai, U. G. Meißner and J. Smejkal, Nucl. Phys. A 954, 17 (2016)

[22] A. Pich, Rep. Prog. Phys. 58, 563 (1995)

[23] G. Ecker, Prog. Part. Nucl. Phys. 35, 1 (1995)

[24] V. Bernard, N. Kaiser, and U. G. Meißner, Int. J. Mod. Phys. E 4, 193 (1995)

[25] P. C. Bruns, M. Mai and U. G. Meißner, Phys. Lett. B 697, 254 (2011)

[26] E. Oset, A. Ramos, Nucl. Phys. A 635, 99 (1998)

[27] U. G. Meißner, U. Raha and A. Rusetsky, Eur. Phys. J. C 35, 349 (2004)

[28] D. N. Tovee et al., Nucl. Phys. B 33, 493 (1971)

[29] R. J. Nowak et al., Nucl. Phys. B 139, 61 (1978)

[30] K. A. Olive et al. (Particle Data Group), Chin. Phys. C 38, 090001 (2014)

[31] G. Passarino and M. J. G. Veltman, Nucl. Phys. B 160, 151 (1979) 


\section{Figure Captions}

Fig. 1 Comparison of poles in the strangeness $S=-1$ and isospin $I=0$ sector. $N E W$ denotes the case calculated from the loop function in Eq. (16), while PRE stands for the case of the loop function in the on-shell factorization approximation in Eq. (13).

Fig. 2 Same as Fig. 1 but for the strangeness $S=-1$ and isospin $I=1$ sector. 
This figure "Fig1a.png" is available in "png" format from: http://arxiv.org/ps/1609.08354v3 
This figure "Fig1b.png" is available in "png" format from: http://arxiv.org/ps/1609.08354v3 
This figure "Fig2.png" is available in "png" format from: http://arxiv.org/ps/1609.08354v3 